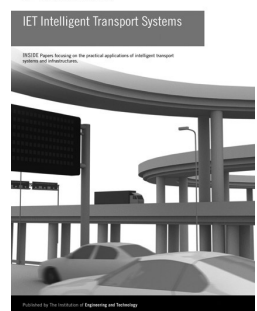

ISSN 1751-956X

\title{
Intelligent transport systems harmonisation assessment: use case of some Spanish intelligent transport systems services
}

\author{
Vicente R. Tomás1, Marta Pla Castells², J. Javier Samper², Francisco R. Soriano² \\ ${ }^{1}$ Ingeniería y Ciencia de los Computadores, Universidad Jaume I, Campus de Riu Sec E-12071. Castellón de la Plana, Spain \\ ${ }^{2}$ Instituto de Robótica y Tecnologías de la Información y las Comunicaciones, Universidad de Valencia, C/ Catedrático José \\ Beltrán, 2, 46980 Paterna, Valencia, Spain \\ E-mail: vtomas@uji.es
}

\begin{abstract}
From the 1980s, new telematic technologies have meant a great evolution in several areas. In the transportation domain, their use has implied the development and implementation of several intelligent transport systems (ITS). However, these deployments were done in an isolated way. Traffic managers, public and private organisations, stakeholders and others have implemented ITS without much perspective, that is, without providing ITS as services for end users. In the last few years, several European Union (EU) funded projects have been dealing with the development of harmonised ITS services. For example, the EasyWay Project is involving most of the European countries (EU member states and others) to deploy harmonised ITS services taking into account the European citizen as the final target. In this study, an introduction of the EasyWay project is made, including the ITS concept services and the deployment guidelines for harmonisation. In November 2012, EasyWay presented a new version of DGs, which were approved with minor editorial changes. An overview on these DGs for the ITS services is presented and two real Spanish road traffic ITS services are analysed.
\end{abstract}

\section{Introduction}

In recent years, the use of new telematic technologies in the transportation domain has made it possible to develop intelligent transport systems (ITS) [1]. ITS can significantly contribute to a cleaner, safer and more efficient transport system contributing to make a better usage of the existing transport infrastructure and to create the necessary momentum to speed up market penetration or rather mature ITS applications and services in Europe.

The European Union has supported several ITS R\&D projects in different frameworks since 1990s, (DRIVE Programme in the old DG-XIII) to the current projects under the Directorate General for Communication Networks, Connect and Technology (DG-CONNECT) [2], DG-MOVE [3] and DG-ENTERPRISE [4] umbrella (European Commission).

In the 1990s, ITS projects were related to the deployment of systems. In that decade, several projects for monitoring systems, communications and incident detections systems [5-9] were deployed to process and obtain real traffic information from the road network and to inform road users. However, all these projects were developed in most of the cases in an isolate way and never following a harmonised European wide approach.

In 2001, in the frame of the TEMPO programme (a multi-annual indicative programme covering the period 2001-2006) a sub-programme for ITS for road was created.
Initially, six Euro-Regional Projects (ARTS, CENTRICO, CORVETTE, SERTI, STREETWISE and VIKING) [10] were deployed as a way to promote a harmonised and synchronised deployment of ITS systems and services on the trans-European road network (TERN). After this initiative, the European platform EasyWay was launched [11]. The main purpose was to foster ITS deployment on the TERN widely. Its activities stretched over a 6-year period, from 2007 until 2012, in two phases. EasyWay was planned as an efficient platform to achieve a coordinated and combined deployment of pan-European ITS services. Two of the EasyWay objectives were to promote future creativity and to create more ambitious targets for future years following the experiences gained by the 'Swedish vision zero'. This vision aimed to achieve a highway system with no fatalities or serious injuries [12]. However, the EasyWay long-term vision is the goal of zero accidents, zero congestion and zero environmental impact.

In line with EasyWay, a new legal framework was adopted in July 2010 to accelerate the deployment of these innovative transport technologies across Europe: the ITS Directive $[13,14]$. This directive is an important instrument for the coordinated and effective deployment and use of ITS in Europe. The directive was addressed to define the compatibility, interoperability and continuity of services as regards the specifications (functional, technical, organisational) of the ITS systems all over Europe. The aim of the directive is to coordinate and accelerate the 
deployment of ITS and to make road transport more sustainable.

In 2011, the European Union White Paper [15] set the targets for 2020 in an attempt to make a positive impact on traffic flow, traffic safety and the environment. The EasyWay project deals with the same targets. Furthermore, EasyWay is providing significant contributions to support the efficient and successful implementation of the ITS action plan across Europe. Mapping EasyWay activities against the ITS directive, demonstrates a very strong correlation with all four priority areas included as development and deployment areas within EasyWay.

The purpose of this paper is to analyse the harmonisation of two ITS Spanish services taking into account the deployment guidelines (DGs) developed in the frame of the EasyWay project. The paper is organised as follows: Section 2 introduces the EasyWay project and the concept of European Core Services and DGs. Section 3 describes two different ITS core services in Spain. Section 4, analyses these two services to assess their degree of compliance with the EasyWay DGs. Finally, Section 5 presents the conclusions and future work.

\section{EasyWay project}

The main scope of EasyWay is to improve safety, mobility and environmental impact on European roads by deploying harmonised ITS services for the European traveller and haulier. EasyWay is formed of national road authorities and operators. Currently it involves 30 European countries (27 Member States plus Andorra, Norway and Switzerland) and more than 150 partners, including the automotive industry, telecom operators and public transport stakeholders [11]. It reaches 450 million people across an area of more than 4 million $\mathrm{km}^{2}$, structured in eight regions. It is important to note that some countries could be part of several regions. For example, Spain is part of SERTI (with France, Italy, Germany and Switzerland) and ARTS (with France and Portugal) regions. The main objectives of the EasyWay programme are [11]: to improve road safety, reduce congestions, improve traffic flows and the environment. Besides its geographical organisation, EasyWay is structured into four main areas when deploying ITS services by focusing on the identification and development of core services. These areas are traffic information, traffic management, freight and logistics and connected information and communication technology (ICT) infrastructure. For each area, a set of ITS core services were identified during the first phase of the project. ITS core services were considered as the most important, required and used in the transportation community. They have been selected from a set of candidate ITS services. ITS core services can also be related between them. For example, the core service 'traffic management plans (TMPs) for corridors and networks' could use other core services such as travel time information or traffic conditions information so as to inform users about the traffic status. Fig. 1 presents the different ITS core services for each area and some relations between them.

\subsection{DGs for ITS core services}

In 2007 after the identification of the ITS core services, the EasyWay partners created a framework for the DGs. The key tool in this process was the implementation and use of common DGs for deploying activities in countries, regions and Europe-wide co-ordinately. For each core service a DG was developed. The purpose of the DGs was to be a reference for deployments on the selected ITS service. The DGs support both the planning and the deployment and provide examples of best practices so as to support the implementation. They also compile the experience of road organisations on the specific item. The purpose of the DGs is to support road organisations in the deployment of harmonised ITS core services. DGs are structured in several domains: traffic information services (TIS-DG), traffic management services (TMS-DG), freight and logistics (F\&L-DG) and supporting DGs [variable message signal (VMS)-DGs, DATEXII-DGs].

A second phase of the elaboration of the DGs started in 2011 [16]. The objective of this phase was to improve the current guidelines by taking to account:

1. A new document structure. Now, the DGs were structured into two parts: part A focused on the recommendations and requirements that have proven to contribute to successful elaboration and that the EasyWay partners have agreed on; and part $\mathrm{B}$ focused on providing more valuable but less

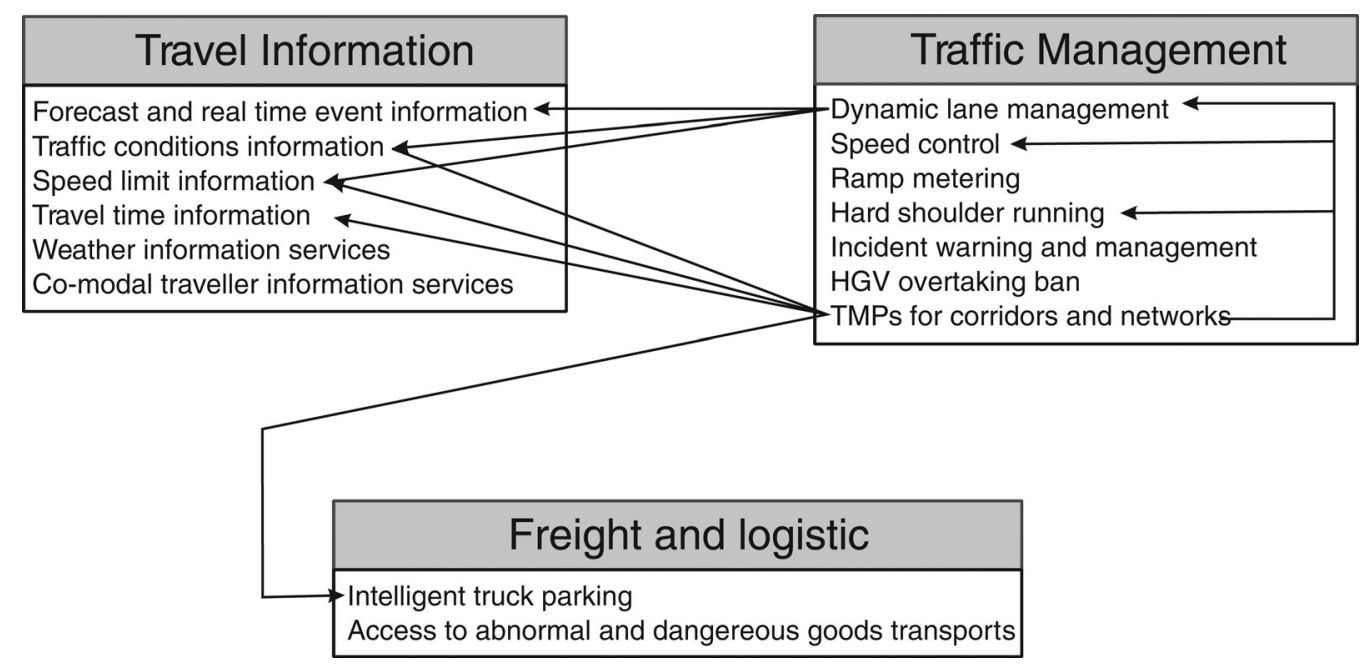

Fig. 1 ITS core services

Only few relations between services are identified 
descriptive information. Therefore additional information was included in this part. For example, regional and national best practice examples, business model aspects such as stakeholder involvement or cost/benefit analysis results and future implementation plans and expected evolution.

2. A set of requirements have been identified for each DG: (i) organisational requirements that focused on the different organisational requirements to deploy the ITS service; (ii) functional requirements that described the functions and the architecture to develop the service; (iii) technical requirements (TR)that described the different TR to deploy the ITS service, (iv) common look and feel that focused on finding an harmonised way so as to present the information to end users and (v) level of services (LoS) requirements.

3. The operating environments (OE) [17]. OE classify the road networks by using a combination of the following key factors: physical characteristics, road section network typology and traffic flow impact and/or road safety concerns. In the frame of EasyWay project, 18 OEs have been used.

4. LoS definition. LoS describes the adequate level of ITS deployment for a concrete road network or segment, depending on the OE to be applied. The LoS is classified in three levels (A, B or C) depending on the ITS service characteristics. For each ITS service, depending on the OE and the LoS, DG presents two indicators: minimum and optimum.

5. The interaction of the service with other ITS services.

In November 2012 all DGs were adopted by all the member states involved in EasyWay, after a deep revision process.

\section{Deployment of ITS core services in Spain}

In Spain Dirección General de Tráfico (DGT), the public road traffic administration in charge of traffic management, has deployed ITS from the 1980s. For example, in 1982 the first monitoring systems based on loops were installed and in 1989 CCTV cameras were added to this system. During that period several ITS services were being developed and upgraded: TMPs, especially focused on coordinated plans [Cross border (CB), emergencies,...], DATEXII, a mechanism to exchange data between organisations, based on the DATEX standards, traffic information systems both on-trip and pre-trip: eTraffic, radio digital system-traffic message channel (RDS-TMC), VMS; TRAZA, a service for controlling the transportation of abnormal goods etc.

One of these services is related to international traffic management: CB TMPs. TMPs were developed in order to cope with road traffic disruptions that called for coordinated actions from the authorities and the different services responsible for road/traffic management on a given road or network. A TMP is the pre-defined allocation of a set of measures to a specific situation in order to inform and control in real-time [18].

Furthermore, DGT provides information to be sent to users by using different systems. These systems are being adapted and upgraded to the new technologies. For example, teletext system used the TV to provide traffic information. At the end of 1999, an RDS-TMC system was deployed. Next, with the general use of mobiles phones, short message service and wireless application protocol services were developed. In 2007 a new real-time traffic information system web-based (http://infocar.dgt.es/etraffic) was developed [19].
The two services have been selected to be evaluated using the DGs because of their relevance. The selected CB TMP is one of the most important services to guarantee traffic flows and road safety when adverse weather situations are produced at international level. eTraffic has been selected because it is the most significant traffic information service (TIS) of DGT.

\subsection{CB TMP for the Atlantic corridor}

In 2000 DGT defined a methodology for the development of TMPs [20]. Following this methodology, several TMPs have been developed [21-23]. These TMPs are focused on big incidences, such as adverse weather situations, incidences on tunnels and emergency situations. In this frame, a coordinated CB TMP was deployed between Spain and France in the Atlantic corridor on the Irun border, from Valladolid to Bordeaux. The traffic situation in the area presents the following characteristics [24]:

- High rates of annual average daily traffic on the border.

- High percentages of heavy good vehicles (HGV). More than $25 \%$ of AADT are related to $\mathrm{HGV}$.

- There are different HGV restrictions on both sides of the border.

- There are several days with adverse weather conditions (more than 64 days of weather problems last winter).

- Several segments of the TMP road network are part of the corridor for summer migrations and important cities are also close to the corridor (Valladolid, Bilbao, San Sebastian, Bordeaux).

This TMP intends to establish how traffic management systems should be used in case of possible weather problems on the road network. It was developed several possible scenarios and the measures to implement each one. The core of the TMP is structured into three information levels: scenarios, measures and actions [20]. The scenario level defines the current status of the incident. The measures level defines the set of procedures necessary for application based on the information of the scenario level. The actions level defines the activities intending to develop each procedure of the measures level.

The scenarios table is composed of four situations in an increasing level of difficulty: from the green level scenario, where the snow is only a forecast, until the black level, where the snow leads the road to being closed. The main measures of this TMP are focused on

- Information services. It includes the exchange between the organisations involved in the TMP and the diffusion of traffic information to end users. These measures include the RDS-TMC alerts, the use of VMS and other information services.

- Activation of storage areas. In red and black scenarios there are traffic restrictions for HGV. Thus, these vehicles must be stored in special rest areas.

- Rerouting. Alternative routes are activated to reroute road users to their destination.

Coordination is fundamental, therefore when one organisation activates the TMP, the exchange of the information protocol is activated to alert about the situation. The protocol includes what information must be shared, when and who are the recipients. 
All traffic control centres (TCCs) involved in the TMP activation have a common view of the scenario and the traffic measures to be deployed. Furthermore, users need harmonised information. The information includes alternative routes, circulation restrictions and the storage area location. The activation of these measures makes use of different TCCs ITS services. For example, the information exchange between TCCs is made not only by email, but also by the standard for traffic information exchange DATEX II [25]. Users are informed by using different on-trip services such as VMS, RDS-TMC, motorway dedicated radio... Furthermore, as the corridor is an international corridor, messages are broadcasted in several languages.

\subsection{Web system to disseminate traffic information: eTraffic}

eTraffic is a TIS, which shows road traffic information via web. It provides information such as:

- Traffic incidences, including civil works on the whole Spanish road network.

- LoS on the main road network.

- Travel times between several predefined origins and destinations.

- Individual monitoring information: CCTV cameras, road traffic sensors, weather road equipment and VMS. It also presents statistics on daily, weekly or monthly traffic flow changes in the selected equipment (traffic and weather sensors).

- Really simply syndication (RSS) subscription service. End users could subscribe to a RSS service to receive updated traffic information.

The information is provided in real-time from different sources of traffic data in Google Maps. Fig. 2 shows an example of the eTraffic application.

The service architecture is based on the general DGT architecture: Capturing automatic data usually happens by monitoring equipment (traffic detectors, such as loops, infrared, CCTV with license plate recognition, ladar, meteo-stations etc). This information is processed by the responsible TCC and is then inserted in the DGT traffic database. Furthermore, other information, which is obtained from different heterogeneous sources, is inserted in the database such as the traffic police, users and toll motorways. Moreover, other traffic organisations send their information via DATEX II. This information is also inserted in the database.

eTraffic is connected to the database to obtain the information users request. It presents several types of traffic-related information; therefore it has been used as best practice for three DGs related to TIS: 'DG02 forecast and real-time event information services', 'DG03-5 traffic condition and travel time information services' and 'DG06 weather information services' [26].

\section{Analysing the degree of compliance of the DGs}

As commented in Section 2.1, the DGs have a set of recommendations and requirements. Each DG has a check list about the requirements that the implemented ITS service (Tables 1 and 2) have to be comply with. These requirements are related to functional (FR), organisational (OR), technical (TR), common look and feel (CL\&FR) and level of services requirements. In order to analyse the degree of compliance of the Spanish ITS services presented, a study has been made about the compliance of the requirement of the two ITS core services exposed in Section 3. The results of this study are presented in the next two subsections.

\subsection{Atlantic CB TMP analysis}

The TMP service for corridors and networks DG (TMSDG07) [27] describes the DGs (elaboration, application and quality control) so as to develop TMPs, especially in CB areas. The objective of this service is the application of traffic management measures in a consistent manner with the aim of increasing the performance of the transport infrastructure.

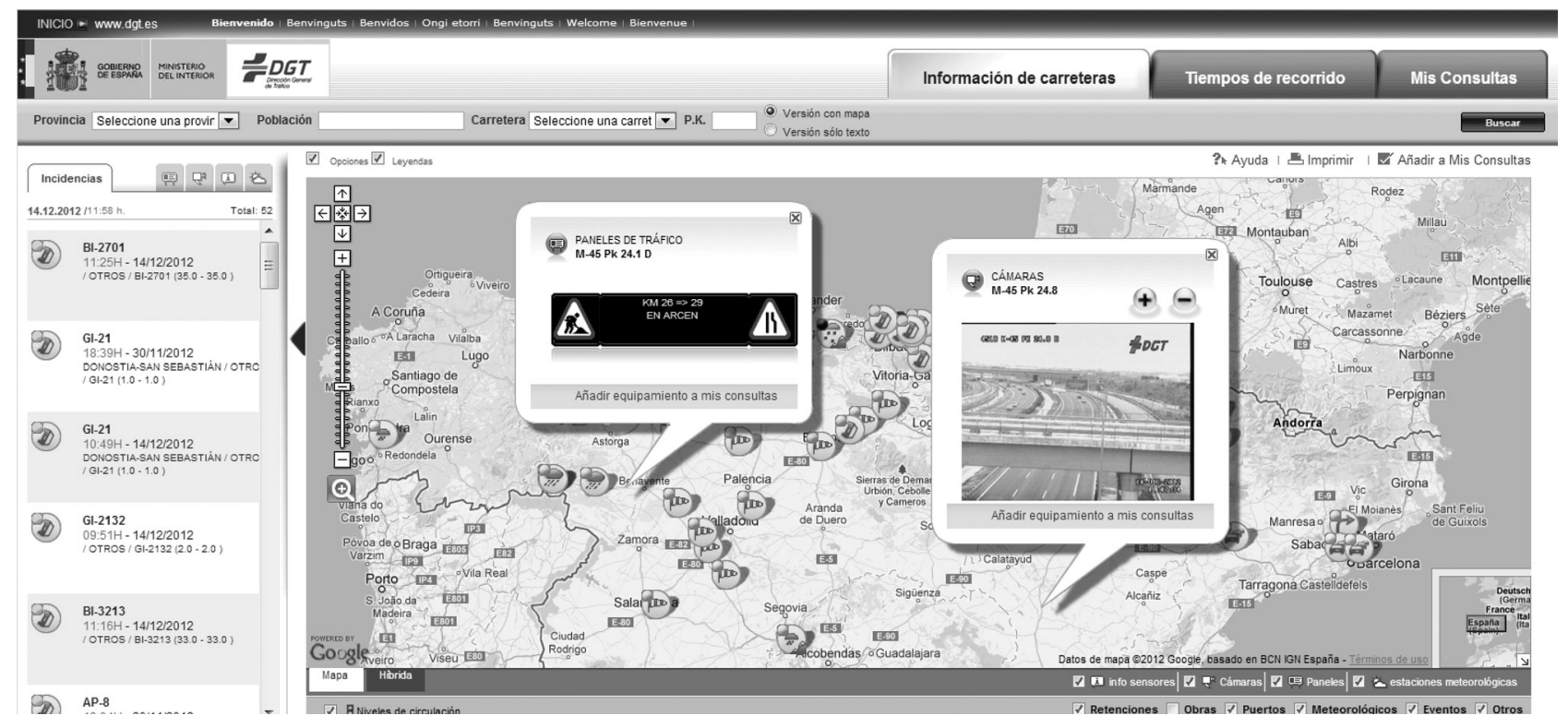

Fig. 2 Spanish eTraffic service 
Table 1 List of functional requirements for TMS-DG07 and the compliance of the presented CB TMP

Functional requirements

TMP elaboration phase

FR1 functional decomposition

FR2 TMP feasibility study must be elaborated

FR3 based on the results of TMP feasibility study a TMP framework must be elaborated and established

FR4 based on the TMP framework, a TMP scenarios document must be delivered

FR5 TMP Scenarios should be profiled in the recommended information structure (DATEXII profiles are not available)

TMP application phase

FR6 functional decomposition based on incident scenario measure

FR7 scenario and measure activation should be provided following the recommendation structure (DATEXII profiles are not available)

FR8 scenario and measure deactivation should be provided following the recommendation structure (DATEXII profiles are not available)

TMP evaluation phase

FR9 existing TMPs must be assessed and periodically adjusted. Hence an evaluation model and an evaluation process must be defined

FR10 TMP evaluation process should compile various information sources

Organisational requirements

Role definition

OR1 all different roles needed in the three phases of the service must be considered and defined (role concept)

OR2 application of the recommended steps for the TMP feasibility study process

OR3 application of the recommended steps for the TMP framework development process

OR4 all necessary organization aspects should be documented and agreed by all involved organisation

OR5 in case of involving private partners, a service level agreement should be developed

OR6 stakeholder involved must agree the operational organisational structures (centralised, decentralised, mixed)

Technical requirements

TR1 communication patterns must be applied

Common look and feel

CL\&FR1 the core message of information provided to end-users should always be consistent whatever the media or the end-user device

CL\&FR2 the display of the sings pictograms on VMS should be in accordance with Vienna Convention

CL\&FR3 for cross-border re-routing arrows the road sign icons identified in the Vienna convention (Annex X, G23, re-routing) must be used

CL\&FR4 for cross-border re-routing signs the road sign icons identified in the Vienna convention (Annex X, G23, re-routing) must be used

CL\&FR5 recommended common structure for the TMP-elaboration document

check

yes

yes

yes

yes

not used

Yes

not used

not use

yes

yes

check

yes

yes

yes

yes

yes

check

yes

check

yes

yes

no

yes
4.1.1 Functional requirements: The functional requirements are focused on the behaviour of the system and serve to identify the services, tasks, functions, relations and so on required to perform the TMP. Functional requirements are structured into three groups: requirements in the elaboration phase, requirements during the execution of the TMP (application phase) and, finally, requirements in the evaluation phase. The elaboration phase requires developing the TMP in several sub-phases (FR1): a feasibility study (FR2) and a TMP framework (FR3). The results of the FR2 and FR3 have to be used to define the TMP scenarios (FR4). The CB TMP presented meets all four requirements. The development of the Atlantic $\mathrm{CB}$ TMP is structured into several phases (FR1). In the first

Table 2 List of functional requirements for TIS-DG02 and the compliance of eTraffic

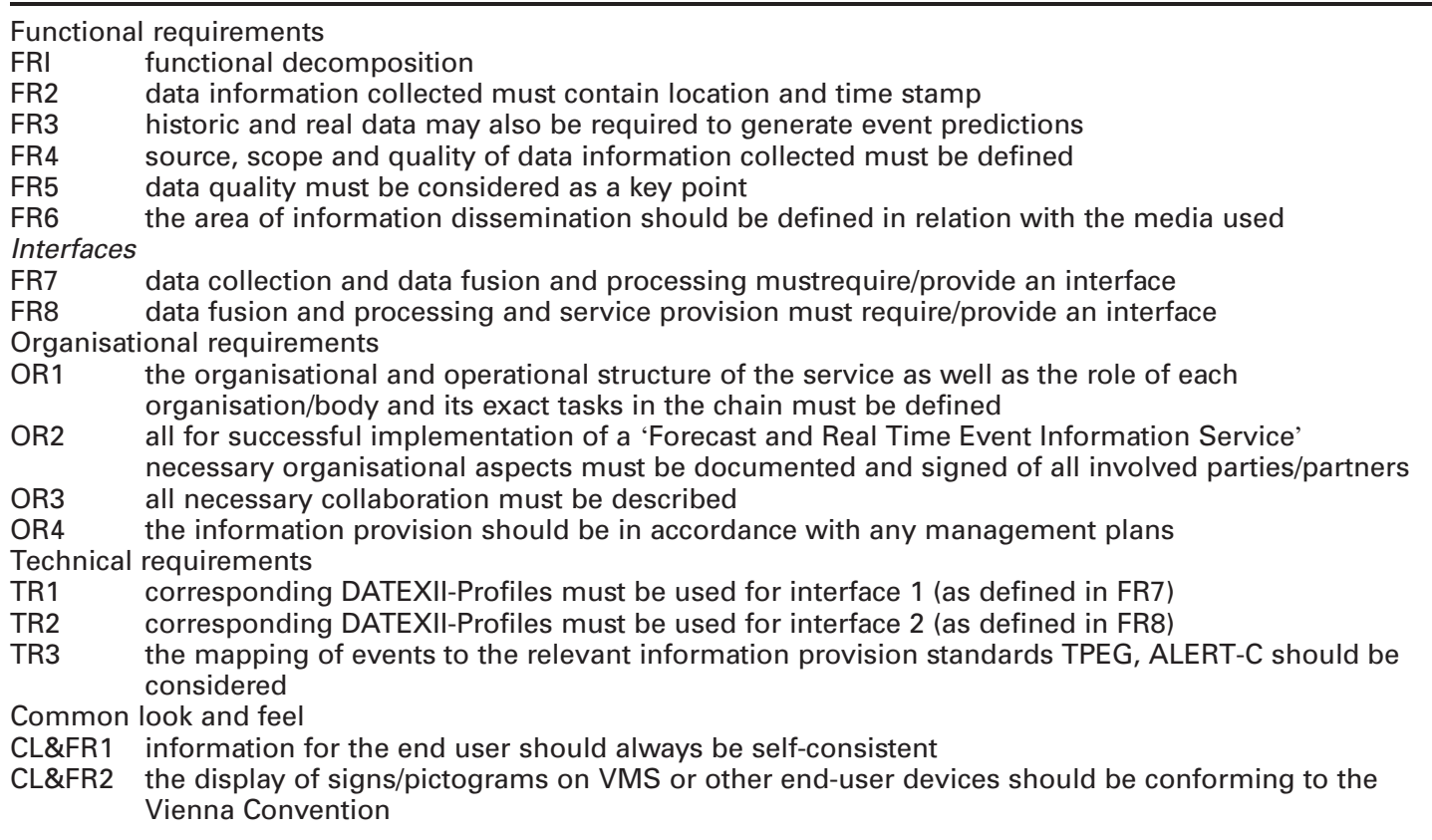

check
yes
yes
yes
yes
yes
yes
yes
yes
check
yes
yes
yes
yes
check
yes
yes
ALERT-C yes
TPEG in process
check
yes
yes




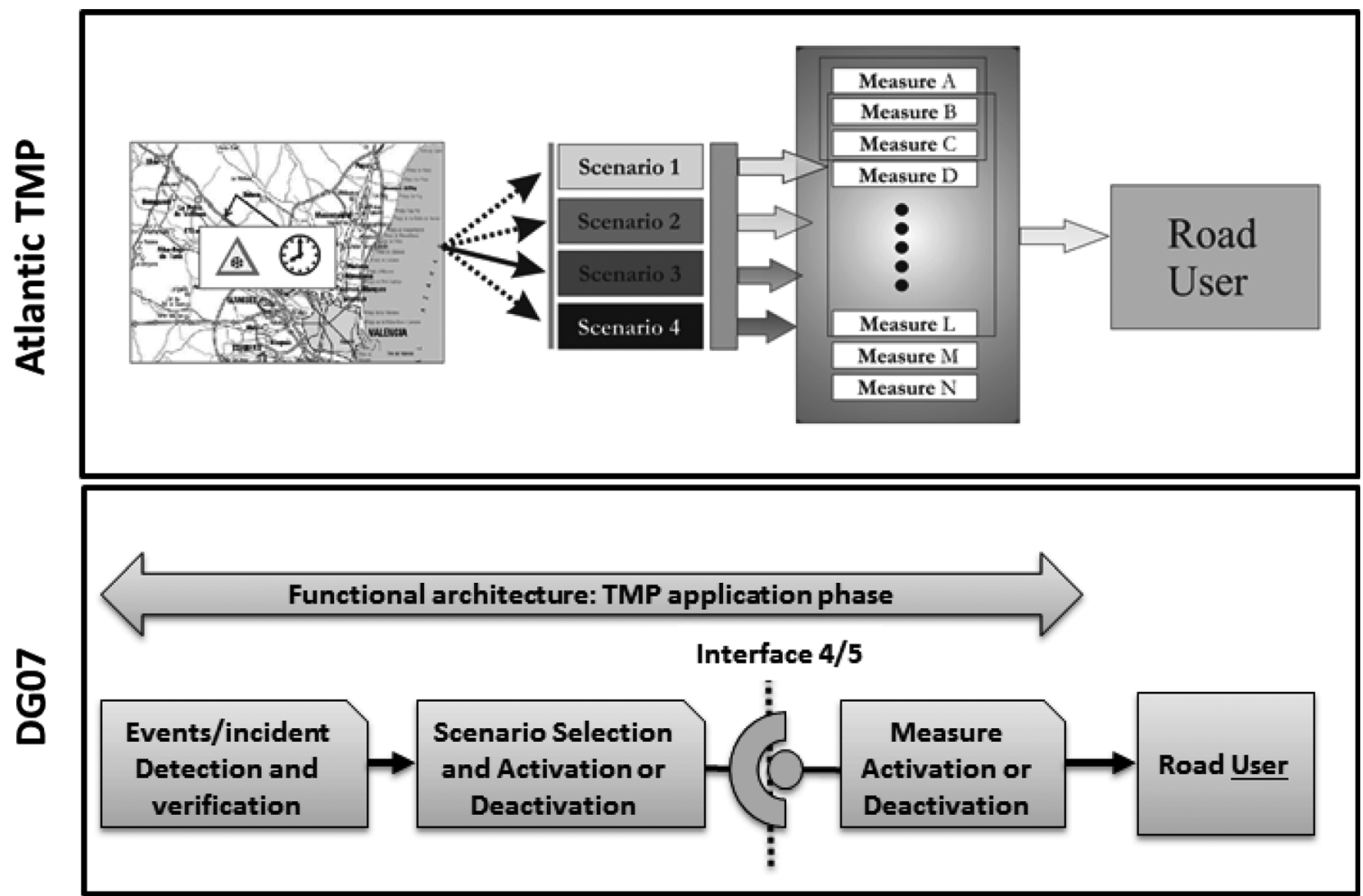

Fig. 3 FR8. Functional decomposition

On the top box, the CB TMP functional decomposition is presented On the bottom, the functional decomposition proposed by DG07 is presented Both approaches are identical

phase, the TCCs involved in the TMP (TCC of Valladolid, TCC of Bilbao and Centre Régional d'Information et de Coordination Routières CRICR of Bordeaux) developed a feasibility study, including road networks, incidents to be included in the TMP and the available ITS services (FR2). Next, using this study, a TMP framework was defined (FR3), which includes all the organisations involved in the TMP and the information exchange mechanism. This mechanism includes not only the information flow between the TCCs and the content, but also the organisations to which information must be sent. In the third phase, using the information of the feasibility study and the TMP framework the scenarios of the TMP were defined (FR4). These depend on the incidents analysed in the feasibility study.

In the application phase, the functional decomposition (FR6) is based on incidents, scenario and measure. This decomposition is the same approach as that of DGT [20]. Therefore FR6 is also reached. Fig. 3 presents the functional decomposition approach, both DGT and DG07.

The evaluation phase happens once the TMP has been activated. The TMP must be assessed and periodically adjusted (FR9). For the evaluation, several sources must be taken into account (FR10). These requirements are also reached. The TCCs responsible meet twice per year. The first meeting is held before the winter period to prepare the activation phase. The second meeting is held after the winter period to analyse the TMP activation and to modify, if needed, not only the scenarios or measures, but also anything related to the TMP (exchange protocol mechanism, road network,...). FR5, FR7 and FR8 are related to DATEX II profiles. However, currently there are not specific DATEX II profiles for TMPs in DGs; so these requirements have not been taken into account.
4.1.2 Organisational requirements: Organisational requirements are aimed at identifying the structures and roles of the organisations involved in the TMP. All organisations and stakeholders involved in the TMP, also known as actors, have to be identified (OR1). This identification has to be carried out in the three phases of the service (elaboration, activation and evaluation).

In the TMP elaboration phase, regarding the feasibility study, OR2 identifies a set of steps to be defined: a) specification of common policy goals and common interests, specification of the involved partners and their scope of responsibility, legal aspects and bases, regulatory framework, identification and analysis of the influence area, among others. OR3 defines the information to be agreed on for the TMP development phase, which involves categorisation of incidents for the activation of the TMP, location codes, development of traffic management strategies, including measures and actions and some others. All organisational aspects of the TMP must be documented and agreed on by the organisations involved in the TMP (OR4). The feasibility study developed in the CB TMP analysed includes all these aspects. All organisations involved in the TMP were identified. Moreover, as there are organisations from different countries (Spain and France), the hierarchical structures and operation processes of action were specified. This study also identifies the TMP road network, including their characteristics and the incidents (or traffic restrictions) that could lead to problems on the other side of the border and the recommendations to be followed in each situation. Thus, OR1, OR2, OR3 and OR4 are reached. OR5 is related to private partners and how this private information has to be included in the TMP. This requirement has not 
been taken into account, because there are not private partners in the TMP.

The organisations involved could have different operational procedures; therefore an agreement of the TMP operational structure has to be made. (OR6). DG proposes three different types: centralised with a TMP coordinator, decentralised or a mixed approach. The presented TMP reaches OR6. It presents a mixed approach with a TMP coordinator. This depends on the organisation responsible where the incident takes place. It is important to note that the coordinator only specifies the measures to be activated and when, not how they have to be activated.

4.1.3 Technical requirement: The communication protocol is fundamental in TMPs, specifically in CB areas. A communication pattern must be applied for the activation or deactivation of a scenario between TMP organisations (TR1). This requirement has been met. The TMP framework identifies the communication exchange protocol. It includes not only the information to exchange, but also the communication procedure between organisations. Currently, the protocol establishes that the information is exchanged by e-mail. However, if there is no acknowledgement of reception in $15 \mathrm{~min}$, the phone and fax are used. A study to use DATEX II as an information exchange protocol is being developed.

\subsubsection{Common look and feel requirement: The} CL\&FR requirements describe the appearance and presentation of the TMP. It is focused not only on end users, but also on the person responsible for the creation, activation and evaluation of the TMP. The core message of the information provided to end-users should always be consistent whatever the media or the end-user device (CL\&FR1) is. This requirement has been met. The information to be exchanged during the TMP activation and evolution has been specified in the specific information exchange measures.

VMS information should be in accordance with prevailing national road codes and, if possible, it must be in line with the EasyWay supporting guideline for variable message signs harmonisation (VMS-DG01) [28] (CL\&FR2). Furthermore, rerouting information should use the rerouting arrows according to the Vienna Convention [29] (CL\&FR3). Spain has adopted the Vienna convention and DGT is the leader of the VMS-DG01. Thus, all the arrows and signals of the TMP follow both the Vienna convention and the VMS-DG01.

For the use of $\mathrm{CB}$ re-routing signs in the itinerary, organisations should use: (i) VMS specific re-routing signs or (ii) static signs in order to mark the rerouting all along the alternative road (CL\&FR4). This requirement has not been met. The specific rerouting signals recommended in the DG are not official in Spain; therefore their use is not possible. Furthermore, road fixed signalisation it not under the competence of DGT; thus, this kind of signalisation is also not possible.

The last requirement, CL\&FR5, is focused on the structure of the document in order to facilitate the comprehension of the TMP document for all the organisations involved in the TMP activation. The CB TMP is only a document; however, it has two versions: one in Spanish and another one in French. The changes made in the document are modified in both documents at the same time, while all organisations upgrade the document. The document index is not the same; however, contents are. Thus, CL\&FR5 has been met.
4.1.5 LoS and operative environment: DGs include LoS for each core European ITS Service. LoS provides the information needed for road operators to ensure that implementations are made in support of European cohesion; it also specifies the steps for the gradual improvement of the service regarding the EasyWay OEs. The LoS defines the recommended level of deployment of the ITS service for a specific road network or segment, depending on the OE to be applied. The OE related to this CB TMP is S1. S1 specifies a motorway corridor or network, with seasonal flow-related impact and possibly safety concerns. The corridor has HGV impact and adverse weather conditions.

For S1 OE the minimum LoS must be:

- Coverage: LoS A. Critical spot coverage. The presented TMP reaches LoS C (optimum). It covers not only the critical spot on the road network, but also all road networks. - Time availability: LoS A. Service periodically ensured during critical periods. The TMP reaches LoS C (more than the optimum). The service is guaranteed $24 \mathrm{~h}, 7$ days per week.

- System availability: LoS A. An only system available. The TMP reaches level C (optimum). Each TCC has several systems to monitor the road network and to inform users and other organisations.

- Consistency: LoS A. Consistent local road user advice along routes. The TMP reaches level C (Optimum). The information is agreed on between the traffic organisations involved in the TMP.

- Level of coordination: LoS A. Knowledge scenario shared between neighbouring regions. The TMP reaches level B (optimum) CB scenario consistency. Currently, new traffic measures are being developed. Once they are implemented the LoS to be reached will be C.

\section{2 eTraffic analysis}

The eTraffic service presents traffic information in real time. It will be analysed with the TIS-DG02, 'forecast and real-time event information services' [26]. This DG is focused on providing information about both expected and unexpected events to road users on the road network (TEN-T). The main objectives are to improve: (i) road safety, (ii) road users' travelling experience and (iii) network efficiency. This type of information services categorises the information in pre-trip and on-trip journey planning. They can lead to changes in departure times, assist the driver to take more effective routing decisions or even change the decision of travelling altogether.

\subsubsection{Functional requirements for eTraffic: A} functional decomposition into sub-functions with the provision of interfaces must be carried out (FR1) so as to guarantee the interoperability in cases that the service is carried out by more than one organisation. These sub-functions are: (i) data collection; (ii) data fusion and processing and (iii) information provision. Fig. 4 presents the functional decomposition of eTraffic and that proposed by DG02.

Each sub-function has to meet some requirements:

- For data collection, all information collected both automatically or manually must contain location code and time stamp (FR2). Moreover, to generate predictions, real data and historic data should be used (FR3). 


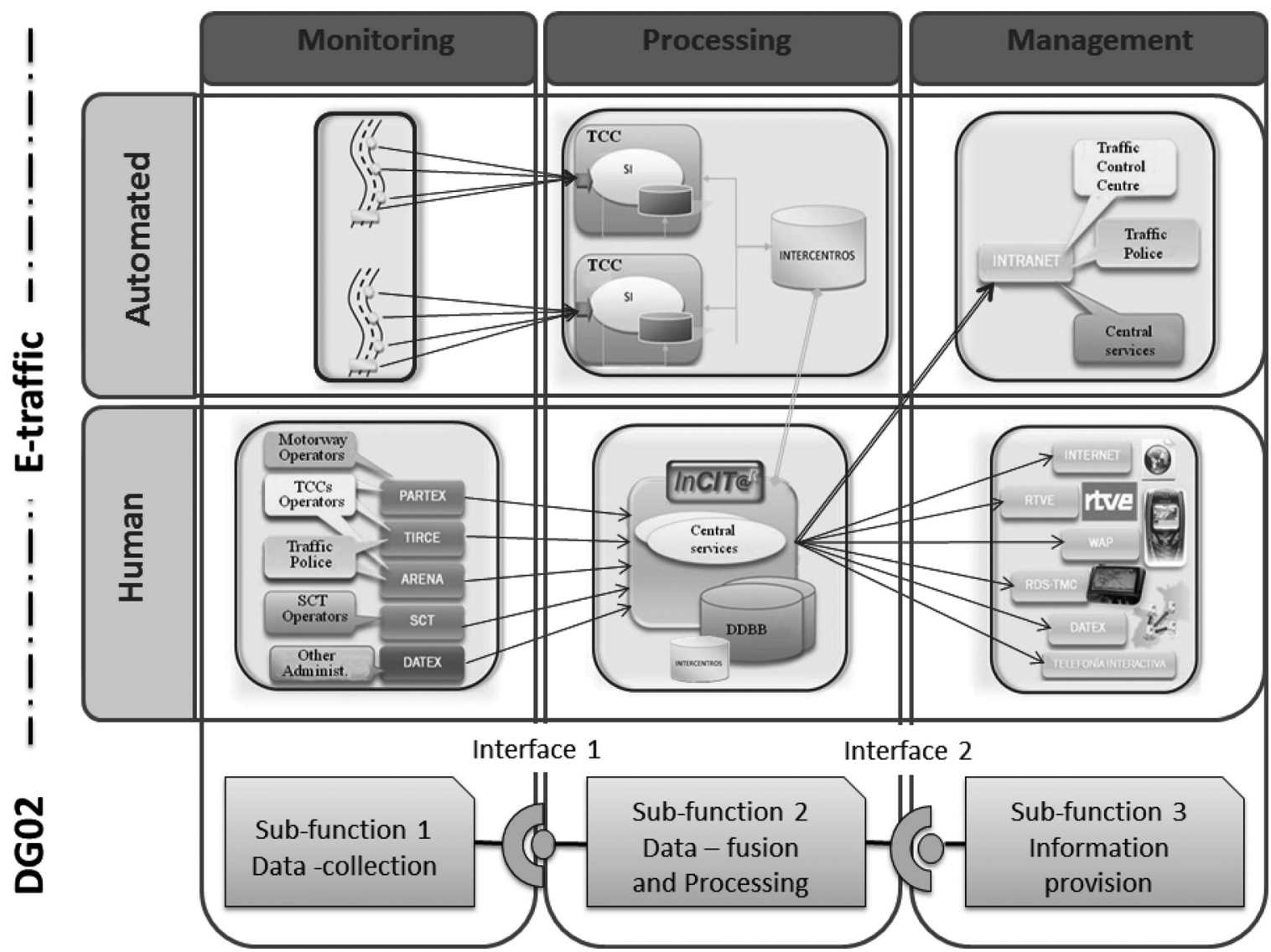

Fig. 4 Functional decomposition of eTraffic

- For data fusion and processing, the source, scope and quality of the data provided must be defined (FR4). Data quality should be considered as a key point (FR5).

- For information provision, the areas and location where the information has to be diffused should be defined (FR6).

Furthermore, there are two requirements regarding the interfaces between the sub-functions. In order to enable interoperability between all the organisations involved, the sub-functions for data collection and fusion and data processing must follow the recommended information structures (FR7); a similar situation must be followed for sub-functions for data fusion and processing and information provision (FR8). eTraffic meets all these eight requirements. All the information inserted in the database and collected (by automatic sensors or manually) has a location code and a timestamp (FR2). DGT forecast processes use not only real-time data information, but also historic values stored in the database (FR3).

Data quality is an important key factor for DGT (FR5). Depending on the source and information content, several data quality processes are executed (FR4). For example, raw data goes through an individual data quality process to be included in the data fusion system. Elaborated data are also analysed with other correlated sensors before including the information in the database.

eTraffic is a TIS that shows the information via web-map browser. Thus, the information presented is related to the zoom that the user selects. All the information available in the zoom window is presented. However, if there is information related to a location out of this window, then it is not visible. Therefore FR6 depends on the user selection.
As commented before, eTraffic shows traffic information from different traffic sources. This process can be done because the information contained in the DGT database is structured following the recommended DATEX II classes. Thus, the requirements FR7 and FR8 are met.

4.2.2 Organisational requirements for eTraffic: As in the previous service, the organisational requirements are focused on the identification of the structures and roles of the organisations involved in the specific service. The structure of services as well as the role of each organisation and its responsibility in the chain must be defined (OR1). Furthermore, the necessary organisational aspects must be documented and agreed on by all the involved parties/ partners so as to establish the co-operation (OR2); all necessary collaboration processes or workflows must be described (OR3).

The organisation responsible of the eTraffic service is DGT. In the information delivery chain of eTraffic only DGT is involved, except for the case when the incident occurs on the road network under the competences of other regional administrations with traffic management competences. DGT has agreements with the two regional traffic authorities in Spain: DTGV (in the Basque Country region) and SCT (in Catalonia region) to publish their traffic information. An information exchange understanding based on DATEX II has been agreed on between the organisations involved. This information exchange mechanism specifies the interfaces and processes so as to update the DGT database in order to include the information that DTGV and SCT provide. Thus, OR1, OR2 and OR3 are met. 
All the information provided by the services should be in accordance with any TMP or traffic measure, which are operated by the road authorities or traffic management centres (OR4). This requirement is also met because the information provided is in the DGT database, where all active traffic management measures are also stored.

4.2.3 TRs for eTraffic: TRs take into account whether or not the service use standards to enable interoperability between the different organisations involved in the forecast and real time event information value chain. In case road operators have to exchange data requiring interoperability between two or more different organisations involved in the forecast and obtain real time event information, the corresponding DATEX II profiles must be used for interface 1 (between data collection and data fusion sub-functions), as defined by FR7 (TR1) and for interface 2 (between data fusion and information provision sub-functions) as defined by FR8 (TR2). Moreover, the information provision should be based on standards (TPEG and ALERT-C are recommended) (TR3). As the information exchanged in eTraffic uses DATEX II, TR1 and TR2 uses the recommended profiles and this information uses the ALERT-C standard to locate the event. However, the TPEG standard is not used.

4.2.4 Common look \& feel (CL\&FR): There are only two requirements regarding CL\&FR in TIS DG02. These requirements are the same as those of TMS DG07. The core message of the information provided to end-users should always be consistent (CL\&FR1) and the VMS information or other devices should be in accordance with prevailing national road codes and, if possible, it must be in line with the VMS-DG01 (CL\&FR2). Both requirements are met. The first requirement is met because the information disseminated by eTraffic is in the database; therefore it is consistent with the situation of the current traffic status. The second requirement is also met. As commented on in Section 4.1.4, Spain has adopted the Vienna convention and DGT leads the VMS-DG01.

4.2.5 LoS and operative environment (OE): eTraffic provides information for the whole Spanish road network. Therefore the LoS is related to the OE which depends on the road network that the user selects. The recommended levels of services are

- User interface. For all OE, except for C1 (sensitive segments), the minimum LoS is to provide information in the official language. This LoS is met. However, for $\mathrm{C} 1$ the minimum LoS recommends providing the information not only in the official language, but also in a common and shared language. eTraffic service does not meet this LoS.

- Neighbour provision. The minimum LoS for all OE, except for $\mathrm{C} 1$, does not require information exchange. The optimum LoS requires information exchange with neighbours only. For $\mathrm{C} 1$, the minimum $\operatorname{LoS}$ is to exchange information with neighbours and the recommended LoS is to extend with neighbours and beyond. All LoS are met. eTraffic uses the information of the DGT Database. This information is exchanged with other organisations (without neighbourhood limits) via DATEX II.

- Information about local and secondary networks. The minimum LoS for all OEs is to provide only information for the TERN. The optimum LoS for all OE, except C1, is to provide information not only on TERN roads, but also on other non-TERN strategic roads. For C1 the LoS is to provide information on all roads that have impact on the TERN roads. All LoS requirements for all OEs are met. The information is provided independently of the road network. In eTraffic, the end user is the one who selects the road network so as to obtain the information. All the information available for the selection is presented.

- Level of detail of the information location. Minimum LoS for R1-R8 (two- and three-lane roads with different characteristics), S1 (motorways without safety concerns or traffic problems), N1 and N2 (corridors) is to inform about the road or the location name where the information is related. The minimum LoS for C1, T1-T4 (motorway that has different characteristics) is to provide the information about the specific route (segment). The optimum LoS for all $\mathrm{OE}$ is to provide the specific location for the information. The optimum LoS is met for all the OE. The information provided by eTraffic is located in the exact point. For example, for traffic data information, the exact position of the equipment station is informed and for travel times, the segment, including origin and destination, is defined.

\section{Conclusions}

The EasyWay project is a great opportunity to deploy harmonised ITS and services across Europe. The project encourages ITS deployment not only at national level, but also at regional and international levels. EasyWay focuses the results not only on road operators but also on end users point of view.

Several ITS core services were identified during the first phase of the project. For each core service a DG is being developed. DGs compile the experience of road organisations regarding the specific ITS service. The purpose of the DG is to support road organisations when deploying harmonised ITS core services.

In this paper, two ITS core services has been presented, one related to strategic traffic management for corridors and networks, that is, a CB TMP for the Atlantic corridor, between Spain and France and the other one for disseminating traffic information using web systems called eTraffic.

TMPs are a useful tool to solve or minimise incidences on the road network, especially when these incidences affect a huge road area network, such as adverse road weather situations. The current TMP for the Atlantic corridor has been presented and analysed by using the TMS-DG07. The TMP meets all mandatory requirements (functional, organisational and CL\&FR). The recommended LoS for the $\mathrm{OE}$ where the road network is located has been also analysed. The results are very positive: not only the minimum level reached in almost all of the cases, but also the optimum. These results indicate that eTraffic is a useful ITS service to plan trips in advance. It shows not only traffic incidences, but also the traffic status. This service has been analysed by using the TIS-DG02 for Forecast and Real Time Event Information Services. The service meets all mandatory requirements of the DGs.

Despite both services meets all mandatory service, some recommended requirements are not currently reached. This situation is being analysed by DGT in order to improve current services and also future developments to reach not only the mandatory, but also the recommended requirements. For example, in the CB TMP service, a deep analysis of the use of DATEX II to improve the information exchange is being done. Furthermore, other ITS services are being analysed using the DGs. 
The action plan, the ITS directive, the EasyWay project share several objectives. The most important one is the harmonisation of ITS service. This harmonisation is focused not only on end users, but also on the deployment of the service. In this way, the DGs developed in EasyWay plays an important role. The DGs are well structured and covers all functional, technical and organisational specifications. Furthermore, it is important to highlight two aspects of DGs: (i) it includes a list of requirements, which explains the definition of service harmonisation and (ii) the LoS for deployment is related to the $\mathrm{OE}$ where the service is installed. However, from our point of view some improvements could be made in the DGs. The service data quality aspects are important, especially when exchange of information is needed. Another issue is related to ICT equipment. Despite the fact that there is a specific chapter in DGs, focused on ICT, a deep analysis of the service requirements in DGs is required, especially taking into account the minimum equipment needed so as to deploy the service.

\section{References}

1 European commission: "White Paper on transport. Roadmap to a single European transport area. Towards a competitive and resource-efficient transport system' (Publications office of the European Union, Luxembourg, 2011)

2 Directorate General Communications Networks, Content and Technology. Available at: http://ec.europa.eu/dgs/connect/index en.htm

3 Directorate General of Mobility and Transport. Available at: http://ec. europa.eu/transport/index_en.htm

4 Directorate General for Enterprise and Industry. Available at: http://ec. europa.eu/enterprise/dg/

5 Keen, K., Hoose, N.: 'INVAID. Integration of computer vision techniques for automatic incident detection'. IEE Colloquium, 1990

6 Ely, S.R.: 'RDS-ALERT: a DRIVE project to develop a proposed standard for the traffic message channel feature of the radio data system RDS'. IEE Colloquium on Car and its Environment, January 1990

7 Michon, J.A.: 'Generic intelligent driver support [Hardcover]' (CRC Press, August, 1993, 1st edn.)

8 Specification of DRIVE Project V1011: 'CARGOES: Integration of dynamic route guidance and traffic control systems' (DRIVE Office, Brussels, 1989)

9 Scemama, G.: 'CLAIRE: a context-free Al based supervisor for traffic control' (Artificial Intelligence Applications to Traffic Engineering, 1994)

10 CONNECT Project: 'Co-ordination and stimulation of innovative ITS activities in Central and Eastern European countries). Available at: http://www.connect-project.org, 2012

11 EasyWay project web page. Available at: www.easyway-its.eu
12 Whitelegg, J., Haq, G.: 'Vision Zero: Adopting a target of zero for road traffic fatalities and serious injuries' (Stockholm Environment Institute, 2006): Available at: http://www.sei-international.org/mediamanager/ documents/Publications/Future/vision_zero_FinalReportMarch06.pdf

13 European commission. Intelligent Transport Systems. 'Action Plan and Directive'. (Publications office of the European Union, Luxembourg, 2010). Available at: http://ec.europa.eu/transport/its/road/action_plan/ action plan en.htm

14 Directive 2010/40/EU of the European Parliament and of the Council of 7 July 2010 on the framework for the deployment of intelligent transport systems in the field of road transport and for interfaces with other modes of transport Text with EEA relevance. Available at: http://eur-lex.europa. eu/LexUriServ/LexUriServ.do?uri=CELEX:32010L0040:EN:NOT

15 WHITE PAPER: 'Roadmap to a single European transport area towards a competitive and resource efficient transport system'. Available at: http://eur-lex.europa.eu/LexUriServ/LexUriServ.do?uri= CELEX:52011DC0144:EN:NOT

16 Coordinator: Stephanie Kleine: 'European ITS core services. EasyWay 2 - deployment guidelines 2012'. Deployment guideline introduction DG-I version 01/02/2000, January 2012

17 Kulmala, R., Innamaa, S., Sundberg, J., Udin, C.: 'Guidance for classifying the EasyWay network into operating environments'. EasyWay 2010-2012. ICT Infrastructures Expert and Studies Group. Operating Environments Task Force. Version: 0.94. 2011

18 Tomás, V.R., Jiménez, A.L., Cambres, C., Soriano, F., Samper, J.J.: 'New technologies to work with traffic management plans'. Traffic Technology International - Annual review, 2003

19 Cirilo, R., Fernández, J., Arbaiza, A., del Campo, S., Niñerola, S.: 'Visualización de eventos de tráfico real basado en SMO'. CIT 2008, 2008

20 Fernandez, F., Berriochoa, L., Ortega, F.: 'Methodology for the creation of traffic management plans in case of snow fall'. Dirección General de Tráfico

21 Jimenez, A.L., Serrano, A.: 'Migrational TMPs: activities in long distance corridors'. I2TERN Euro Conf., Dublin, 2005

22 Belda, E., Tomás, Vicente R.: 'ITS for emergency resolution'. 11th World Congress on ITS, Nagoya, Japan, 2004

23 Alberto, S., Federico, F., Enrique, B., Vicente, R.T., María, H.: 'A methodology to improve coordination in tunnels incidents management'. 15th World Congress on ITS, New York, EE.UU, 2008

24 Soriano, F., Rodriguez, M.A., Aizpura, J.: 'Cross Border Traffic Management in the Atlantic Corridor'. I2tern 2008. 1st European EasyWay Conf., 2008

25 European Commission Directorate General for Transport and Energy: 'DATEX II V1.0 USER GUIDE 2006'. Annex IX and Annex 10 of ECE/TRANS/WP.1/119/Rev.2, 27 May 2010

26 EasyWay Project: 'TIS-DG02. - Forecast and real time event information services' Deployment Guideline, 2012

27 EasyWay Project: 'TMS-DG07. - Traffic management plan service for corridors and networks' Deployment Guideline, 2012

28 EasyWay Project: 'VMS-DG01. - Variable message signs harmonisation. Principles of VMS design' Deployment Guideline, 2012

29 UNECE (1968/1995). Convention on road signs and signals, E/ CONF.56/17/Rev.1/Amend.1. Available at: http://www.unece.org/ trans/main/welcwp1.html 